\title{
Predictors of deep-vein thrombosis in subarachnoid hemorrhage: a retrospective analysis
}

\author{
Federico Geraldini ${ }^{1}$ (1) - Alessandro De Cassai ${ }^{1} \cdot$ Christelle Correale $^{1} \cdot$ Giulio Andreatta $^{1} \cdot$ Marzia Grandis $^{1}$. \\ Paolo Navalesi ${ }^{1}$ - Marina Munari ${ }^{1}$
}

Received: 3 April 2020 / Accepted: 9 June 2020 / Published online: 23 June 2020

(C) Springer-Verlag GmbH Austria, part of Springer Nature 2020

\begin{abstract}
Background Subarachnoid hemorrhage is a severe subtype of hemorrhagic stroke, and deep-vein thrombosis is a frequent complication detected in these patients. In addition to other well-established risk factors, the early activation of coagulation systems present in patients with subarachnoid hemorrhage could potentially play a role in the incidence of deep-vein thrombosis. This study aims to identify possible predictors for deep-vein thrombosis related to subarachnoid hemorrhage.

Methods We conducted a retrospective cohort study on patients with a diagnosis of subarachnoid hemorrhage who presented to our institution between 1 January 2014 and 1 August 2018. We reviewed electronic medical records and analyzed several parameters such as Fisher scale, World Federation of Neurosurgical Surgeons scale, aneurysm site, surgical or endovascular treatment, decompressive craniectomy, vasospasm, infection (meningitis and pneumonia), presence of motor deficit, length of stay in the ICU, length of hospital stay, number of days under ventilator support, d-dimer at hospitalization, and the time to thromboprophylaxis (days).

Results The univariate analysis showed that intraparenchymal cerebral hemorrhage, d-dimer at hospitalization, the time to thromboprophylaxis, motor deficit, and aneurysm located at the internal carotid artery were statistically significant factors. Intraparenchymal cerebral hemorrhage (OR 2,78 95\% CI 1.07-7.12), motor deficit (OR 3.46; 95\%CI 1.37-9.31), and d-dimer at hospitalization (OR $1.00295 \%$ CI 1.001-1.003) were demonstrated as independent risk factors for deep-vein thrombosis. Length of hospital stay was also found to be significantly longer in patients who developed deep-vein thrombosis ( $p$ value 0.018 ). Conclusion Elevated d-dimer level at the time of hospitalization, motor deficit, and the presence of an intraparenchymal hemorrhage are independent risk factors for deep-vein thrombosis. Patients with DVT also had a significantly longer hospital stay. Even though further studies are needed, patients with elevated d-dimer at hospitalization and intraparenchymal cerebral hemorrhage may benefit from a more aggressive screening strategy for deep-vein thrombosis.
\end{abstract}

Keywords Deep-vein thrombosis $\cdot$ Subarachnoid hemorrhages $\cdot$ Retrospective study $\cdot$ Risk assessments $\cdot$ D-dimer $\cdot$ Intracerebral hemorrhage

\section{Abbreviations}

ACA Anterior cerebral artery

ACoA Anterior communicating artery

DCI Delayed cerebral ischemia

DVT Deep-vein thrombosis

This article is part of the Topical Collection on Neurosurgical intensive care

Federico Geraldini federico.geraldini@gmail.com

1 UOC Anaesthesia and Intensive Care Unit, Department of Medicine-DIMED, University of Padua, Via Giustiniani 1, Padova, Italy

$\begin{array}{ll}\text { EMR } & \text { Electronic medical records } \\ \text { ICA } & \text { Internal carotid artery } \\ \text { ICH } & \text { Intraparenchymal cerebral hemorrhage } \\ \text { ICU } & \text { Intensive care unit } \\ \text { IVH } & \text { Intraventricular hemorrhage } \\ \text { MCA } & \text { Middle cerebral artery } \\ \text { PCA } & \text { Posterior cerebral artery } \\ \text { PT } & \text { Pharmacological thromboprophylaxis } \\ \text { SAH } & \text { Subarachnoid hemorrhage } \\ \text { SM } & \text { Sine materia } \\ \text { TF } & \text { Tissue factor } \\ \text { TFPI } & \text { Tissue factor plasminogen inhibitor } \\ \text { WFNS } & \text { World Federation of Neurosurgical Surgeons scale }\end{array}$




\section{Introduction}

Subarachnoid hemorrhage (SAH) is a severe subtype of hemorrhagic stroke associated with a high risk of mortality and disability [4].

The connection between the arterial circulation and the subarachnoid space causes a rapid increase in intracranial pressure, possibly leading to death within minutes [5].

Survivors from acute hemorrhage may develop delayed cerebral ischemia (DCI), which was historically related to vasospasm, but recent findings suggest that its pathogenesis is more complex and still not completely understood [16]. Prompt activation of the coagulation system could play a role in SAH pathogenesis contributing to microthrombosis and DCI [18].

Besides neurological complications, venous thromboembolism, which encompasses deep-vein thrombosis (DVT) and pulmonary embolism, is not an uncommon evenience in these patients (incidence ranging from 3.4 to $24 \%$ ) and it is associated with an increased mortality and morbidity rate $[7$, $9,12,15]$. Previously established risk factors are increased age, black race, male sex, paralysis, neurologic disorders, and coagulopathy [7]. We hypothesized that in addition to well-established risk factors, the forceful early activation of the coagulation system present in patients with SAH could potentially relate with the risk of DVT.

This study aims to evaluate early predictors of deep-vein thrombosis in SAH patients, in order to identify patients which could benefit from a more aggressive screening strategy.

\section{Methods}

We conducted a retrospective cohort study on patients suffering from SAH. The study protocol was in accordance with the 1964 Declaration of Helsinki and its later amendments. Informed consent was obtained whenever possible from each participant or its legal representative. The study protocol was reviewed and approved by the Ethics Committee for Clinical Research of the Padova Medical Hospital.

A retrospective review of electronic medical records (EMR) of patients with SAH who presented at our institution (University Hospital of Padova) from 1 January 2014 to 1 August 2018 was performed in order to obtain demographic information such as age, gender, and past medical history. Past medical history included history of hypertension, diabetes, use of tobacco, obesity (BMI $>30 \mathrm{~kg} / \mathrm{m}^{2}$ ), and chronic kidney disease (collected by ICD-10 codes). These data were used as preliminary group comparison to evaluate group homogeneity. Inclusion criteria were age $>18$ years and radiological and clinical diagnosis of acute SAH. Exclusion criteria were prior use of anticoagulants or hepatic dysfunction with coagulopathy.
Secondly, we reviewed EMR in order to obtain SAHrelated data such as Fisher scale, World Federation of Neurosurgical Surgeons (WFNS) scale, aneurysm site (anterior cerebral artery $[\mathrm{ACA}]$, anterior communicating artery $[\mathrm{ACOA}]$, internal carotid artery [ICA], middle cerebral artery $[\mathrm{MCA}]$, posterior cerebral artery [PCA], sine materia [SM]), intraventricular hemorrhage (IVH), intraparenchymal cerebral hemorrhage (ICH), surgical or endovascular treatment, decompressive craniectomy, vasospasm, $\mathrm{d}$-dimer $(\mathrm{mcg} / \mathrm{L})$ at hospitalization, time to thromboprophylaxis(days), total days under mechanical ventilation (days), infections (e.g., pneumonia, meningitidis), motor deficit (plegia or paresis), length of hospitalization, ICU length of stay(days), and anamnestic factors such as active smoking, obesity, diabetes, hypertension, and age. These factors were analyzed as possible predictors of DVT related to SAH.

Moreover, we searched in EMR for DVT events in the 90 days following SAH. Venous duplex ultrasound was usually done according to our local clinical practice at least once during the first 2 weeks, and further DVT screening was based on clinical suspicion.

D-dimer was analyzed using an immunoturbidimetric assay applied on a Sysmex CS-5100 System using SCLAVO reagents. The upper limit for normal values has been defined by our laboratory at $250 \mathrm{mcg} / \mathrm{L}$ and is increased by $50 \mathrm{mcg} / \mathrm{L}$ for every decade of age after 50 years.

Data for each continuous variable was analyzed for a normal distribution using the Shapiro-Wilk test. Results for continuous variables with normal distributions were expressed as mean (standard deviation) values; those with non-normal distributions were expressed as median (firstthird quartile) values. Analysis of data with a normal or a non-normal distribution was performed using the two-tail Student's $t$ test and the Mann-Whitney $U$ test, respectively. The results for analyses of categorical variables were reported as numbers (percentages) and were compared between groups using the chi-square test or the Fisher's exact test when appropriate.

To determine the relationships between the dependent categorical variable (i.e., DVT) and one or more independent categorical variables (i.e., DVT predictors), we performed a multiple logistic regression analysis to calculate odds ratios (ORs) with 95\% confidence intervals (CIs), performing backward and forward stepwise regression to select the best model.

The area under the receiver operating characteristic (ROC) curve was used to examine the predictive performance of DVT predictors. The sensitivity and specificity plots were used to determine the cut-off points for DVT prediction. Days free from DVT were estimated by using Kaplan-Meier analysis.

All statistical analyses were performed using $\mathrm{R}$ version 3.4.0 (2017-04-21). $P$ values $<0.05$ were considered to indicate a statistically significant result. 


\section{Results}

A total of 170 patients were admitted at our institution with a diagnosis of SAH between 1 January 2014 and 1 August 2018. Median age was 59.5 (50-70), 56 were male, and 114 were female. Aneurysms locations were distributed as follows: ACA 13 (7.6\%), AcoA 57 (33.5\%), ICA 19 (11.2\%), MCA 32 (18.8\%), PCA 37 (21.7\%), and SM 12 (7.0\%).

Forty-three patients developed a clinically evident infection requiring antibiotic therapy: 42 developed pneumonia with typical lung infiltrates and a single patient developed a meningitidis with positive cerebrospinal fluid cultures.

Twenty-nine patients (17\%) developed DVT (day of diagnosis 10 [7-17]). No episodes of pulmonary embolism were detected. Homogeneity across DVT group and non-DVT group is reported in Table 1. Median time to DVT diagnosis was 10 days. Among the patients with confirmed DVT, 15 developed proximal vein thrombosis, whereas 14 developed distal DVT of the soleal veins. Among the distal DVT groups, further screening reported no cases of proximal progression after the initiation of anticoagulation therapy.

Univariate analysis of DVT predictors related to SAH identified a statistically significant difference between groups for the following variables: aneurysm located in ICA, ICH, ddimer at the time of hospitalization, time to pharmacological thromboprophylaxis (PT), and motor deficit (Tables 1 and 2).

However, logistic regression identified only ICH (OR 2.78; 95\% CI 1.07-7.12), d-dimer at hospitalization (OR 1.002; 95\% CI 1.001-1.003), and motor deficit (OR 3.46; 95\%CI 1.37-9.31) as independent risk factors for DVT (Table 3).

The reported ROC curve for d-dimer level at the time of hospitalization had an area under the curve (AUC) of 0.678 (95\%CI: 0.5736-0.782) with a Youden index identified at $687 \mathrm{mcg} / \mathrm{L}$ (Fig. 1); a higher AUC was found evaluating the combined ROC curve for both d-dimer and ICH (AUC 0.714;

Table 1 Univariate analysis of classic predictors of deep-vein thrombosis. $D V T$ deep-vein thrombosis, $B M I$ body mass index, $C K D$ chronic kidney disease

\begin{tabular}{llll}
\hline & DVT $(n=29)$ & No DVT $(n=141)$ & $p$ value \\
\hline Male sex & $10(34.50 \%)$ & $46(32.60 \%)$ & 0.846 \\
Age (years) & $63(53-72)$ & $58(48-69)$ & 0.155 \\
Cancer & $0(0.00 \%)$ & $2(1.40 \%)$ & 0.518 \\
Diabetes mellitus & $2(6.90 \%)$ & $9(6.40 \%)$ & 0.918 \\
Liver disease & $0(0.00 \%)$ & $3(2.10 \%)$ & 0.428 \\
BMI $>30 \mathrm{~kg} / \mathrm{cm}^{2}$ & $0(0.00 \%)$ & $2(1.40 \%)$ & 0.518 \\
Hypertension & $16(55.20 \%)$ & $58(41.10 \%)$ & 0.165 \\
CKD $(\%)$ & $0(0.00 \%)$ & $1(0.70 \%)$ & 0.649 \\
Tobacco use & $6(20.70 \%)$ & $32(22.70 \%)$ & 0.813 \\
\hline
\end{tabular}

*Statistically significant results
Table 2 Univariate analysis of possible predictors of deep-vein thrombosis linked to subarachnoid hemorrhage.

\begin{tabular}{llll}
\hline & DVT $(n=29)$ & No DVT $(n=141)$ & $p$ value \\
\hline ICA & $0(0.00 \%)$ & $18(13.47 \%)$ & $0.046^{*}$ \\
MCA & $7(24.13 \%)$ & $25(17.73 \%)$ & 0.467 \\
ACA & $3(10.34 \%)$ & $10(7.09 \%)$ & 0.388 \\
ACoA & $12(41.37 \%)$ & $48(34.04 \%)$ & 1.000 \\
CP & $6(20.68 \%)$ & $30(21.98 \%)$ & 0.437 \\
SM & $1(3.44 \%)$ & $10(7.80 \%)$ & 0.693 \\
ICH & $11(37.90 \%)$ & $27(19.10 \%)$ & $0.027^{*}$ \\
IVH & $14(48.30 \%)$ & $71(50.40 \%)$ & 0.838 \\
Vasospasm & $9(31.00 \%)$ & $41(29.10 \%)$ & 0.833 \\
Surgical clipping & $14(48.30 \%)$ & $45(31.90 \%)$ & 0.091 \\
Dec craniectomy & $5(17.20 \%)$ & $12(8.5 \%)$ & 0.153 \\
WFNS scale & $2(1-5)$ & $3(2-5)$ & 0.996 \\
Fisher scale & $4(3-4)$ & $4(3-4)$ & 0.168 \\
D-dimer at hosp (mcg/L) & $965(488-2166)$ & $543(266-1107)$ & $0.002^{*}$ \\
PT start (days) & $4(3-5)$ & $3(2-4)$ & $0.010^{*}$ \\
Ventilation days (days) & $14.17 \pm 11.46$ & $11.06 \pm 12.31$ & 0.196 \\
Infection (\%) & $8(27.59 \%)$ & $35(24.82 \%)$ & 0.755 \\
Motor deficit (\%) & $17(58.62 \%)$ & $47(33.33 \%)$ & $0.010^{*}$ \\
\hline
\end{tabular}

$I C A$ internal carotid artery, $M C A$ middle cerebral artery, $A C A$ anterior cerebral artery, $A C o A$ anterior communicating artery, $C P$ posterior cerebral artery, $S M$ sine materia, $I C H$ intraparenchymal cerebral hemorrhage, $I V H$ intraventricular hemorrhage, WFNS World Federation of Neurological Surgeons, $P T$ pharmacologic thromboprophylaxis

*Statistically significant results

CI: 0.614-0.810) and d-dimer, ICH, and motor deficit (AUC: 0.718; CI 0.618-0.815) (Fig. 2).

Kaplan-Meier curves for both a d-dimer level of $687 \mathrm{mcg} / \mathrm{L}$ ( $p$ value 0.006 ) and ICH ( $p$ value 0.003 ) are reported in Figs. 3 and 4 respectively.

Although ICU length of stay was not different among the groups $(20.00 \pm 15.19$ vs $16.57 \pm 15.99 ; p$ value 0.278$)$, patients with DVT had a statistically significant longer hospital stay ( $38.62 \pm 20.65$ vs $28.27 \pm 21.42 ; p$ value 0.018$)$.

\section{Discussion}

In our study, we found that in patients with $\mathrm{SAH}$, the elevation of d-dimer levels at the time of hospitalization, the presence of motor deficit, and the presence of an ICH are independent risk factors for DVT.

Length of hospital stay was also found to be significantly increased in patients with DVT, whereas length of ICU stay was not significant.

Time on ventilatory support was used as a surrogate marker for immobility and sedation and has been previously associated with DVT in several studies [10]. On average, our 
Table 3 Logistic regression and fitted model of possible predictors of deep-vein thrombosis linked to subarachnoid hemorrhage.

\begin{tabular}{|c|c|c|c|c|c|c|}
\hline & \multicolumn{3}{|c|}{ Logistic regression } & \multicolumn{3}{|c|}{ Fitted model } \\
\hline & OR & $95 \% \mathrm{CI}$ & $p$ value & OR & $95 \% \mathrm{CI}$ & $p$ value \\
\hline $\mathrm{ICA}(\%)$ & $6.26 * 10^{-8}$ & $3.5 .83 * 10^{-50}-2.68 * 10^{24}$ & 0.990 & & & \\
\hline $\operatorname{MCA}(\%)$ & 0.99 & $0.18-6.35$ & 0.997 & & & \\
\hline $\operatorname{ACA}(\%)$ & 1.15 & $0.34-4.32$ & 0.935 & & & \\
\hline $\mathrm{ACoA}(\%)$ & 1.40 & $0.29-8.49$ & 0.688 & & & \\
\hline $\mathrm{CP}(\%)$ & 1.25 & $0.23-8.32$ & 0.802 & & & \\
\hline $\operatorname{SM}(\%)$ & 0.65 & $0.02-9.61$ & 0.769 & & & \\
\hline $\mathrm{ICH}(\%)$ & 2.59 & $0.81-8.39$ & 0.106 & 2.78 & $1.07-7.12$ & $0.032 *$ \\
\hline $\operatorname{IVH}(\%)$ & 0.99 & $0.33-2.99$ & 0.986 & & & \\
\hline Vasospasm (\%) & 1.11 & $0.38-3.05$ & 0.840 & & & \\
\hline Surgical clipping (\%) & 0.49 & $0.16-1.42$ & 0.194 & & & \\
\hline Dec craniectomy $(\%)$ & 1.65 & $0.34 .-7.75$ & 0.520 & & & \\
\hline WFNS scale & 0.71 & $0.43-1.10$ & 0.147 & 0.73 & $0.50-1.02$ & 0.086 \\
\hline Fisher & 0.97 & $0.001-1.11$ & 0.819 & & & \\
\hline D-dimer at hosp (mcg/L) & 1.003 & $1.001-1.005$ & $0.028^{*}$ & 1.002 & $1.001-1.003$ & $0.042 *$ \\
\hline PT start (days) & 1.16 & $0.90-1.05$ & 0.226 & & & \\
\hline Ventilation days (days) & 1.004 & $0.95-1.05$ & 0.864 & & & \\
\hline Infection $(\%)$ & 0.71 & $0.20-2.44$ & 0.597 & & & \\
\hline Motor deficit (\%) & 6.3 & $1.77-24.2$ & $0.005^{*}$ & 3.46 & $1.37-9.31$ & $0.010 *$ \\
\hline
\end{tabular}

$I C A$ internal carotid artery, $M C A$ middle cerebral artery, $A C A$ anterior cerebral artery, $A C O A$ anterior communicating artery, $C P$ posterior cerebral artery, $S M$ sine materia, $I C H$ intraparenchymal cerebral hemorrhage, $I V H$ intraventricular hemorrhage, WFNS World Federation of Neurological Surgeons, $P T$ pharmacologic thromboprophylaxis

*Statistically significant results

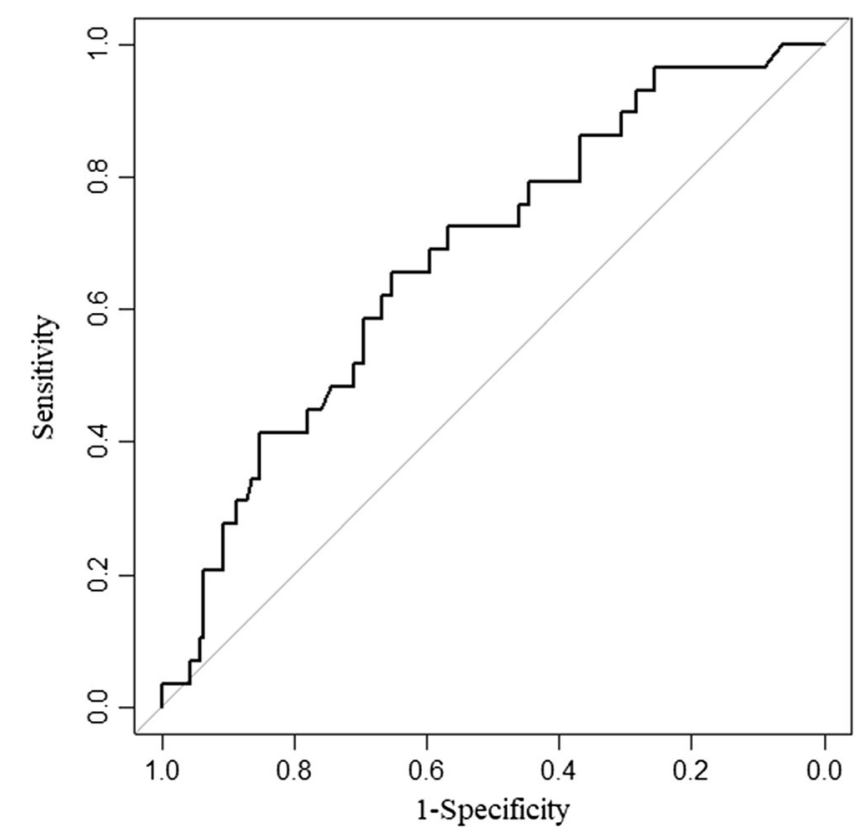

Fig. 1 Receiving operator curve for d-dimer level at hospitalization

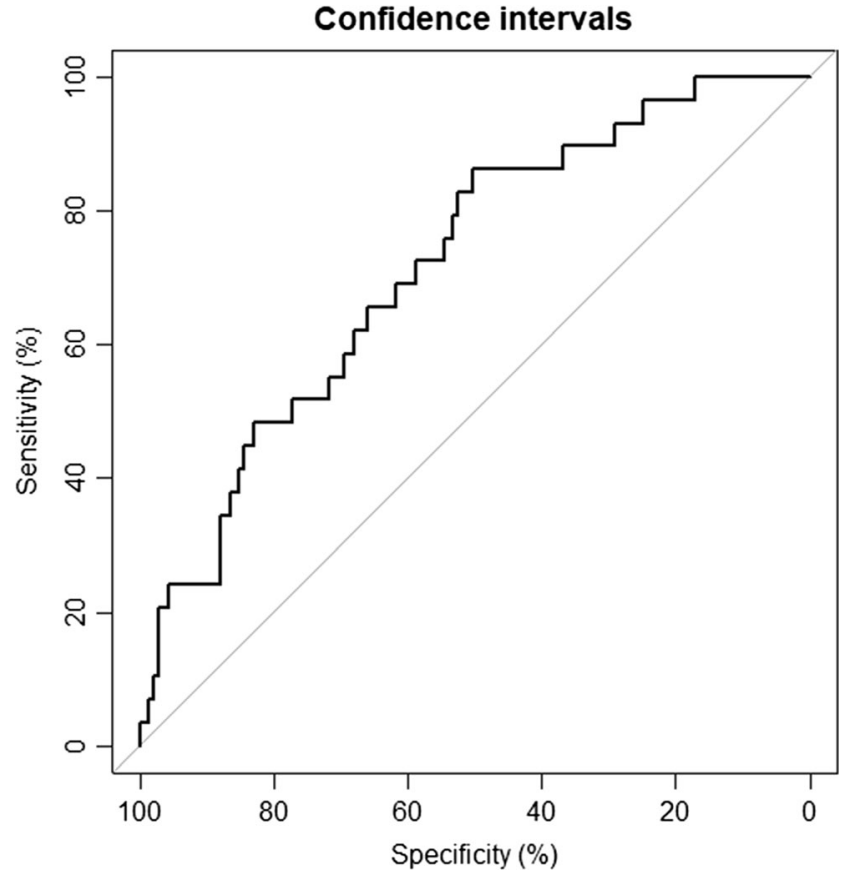

Fig. 2 Combined ROC curve for d-dimer, motor deficit, and ICH 


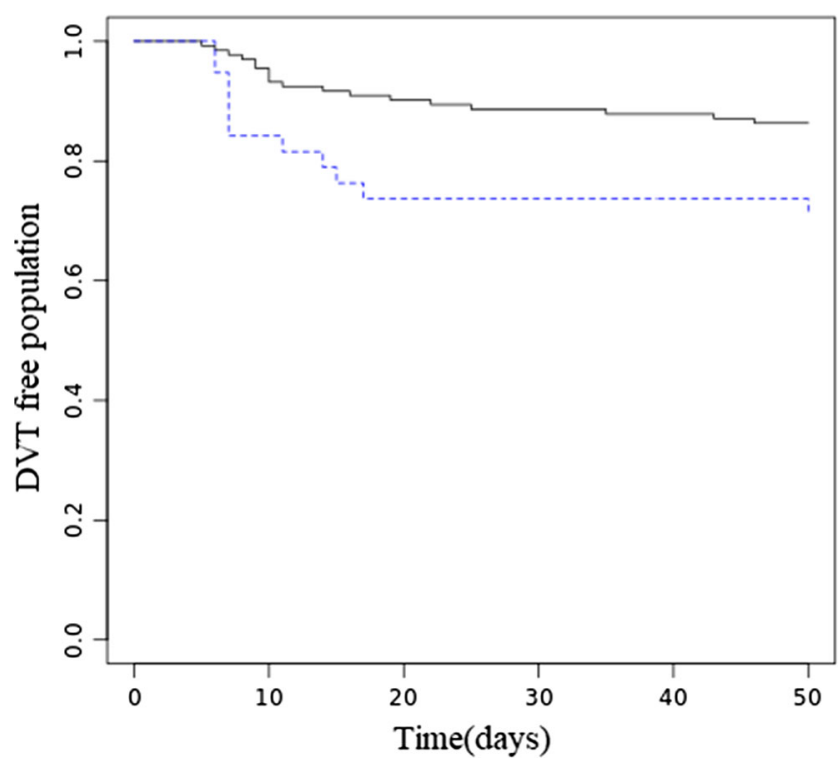

Fig. 3 Kaplan-Meier curve for deep-vein thrombosis and intraparenchymal cerebral hemorrhage ( $p$ value 0.003 ). Black continuous line represents population without intraparenchymal cerebral hemorrhage while blue dotted line represents population with intraparenchymal cerebral hemorrhage

patients were ventilated for 11.6 days before extubation or spontaneous breathing with tracheostomy. There was no statistically significant difference in the number of days on ventilatory support between the DVT group and the non-DVT group.

The management of DVT and its pharmacological prophylaxis is challenging in the context of acute intracranial bleeding. Furthermore, patients with SAH often require the placement of an external ventricular drain and/or a craniectomy in

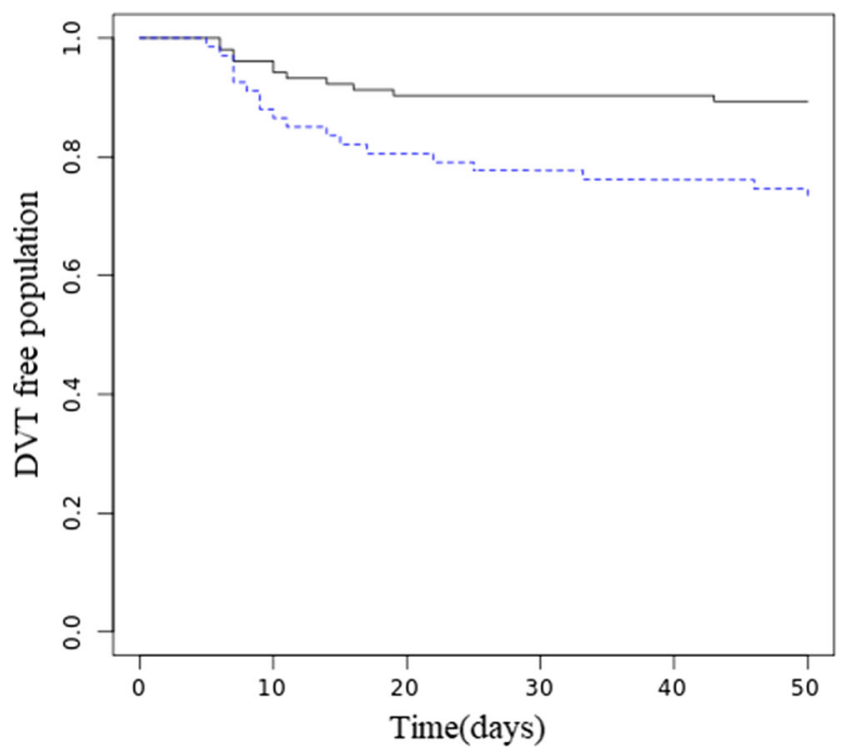

Fig. 4 Kaplan-Meier curves for deep-vein thrombosis ( $p$ value 0.006). Black continuous line represents population with d-dimer below $687 \mathrm{mcg} / \mathrm{L}$ while blue dotted line represents population with d-dimer above $687 \mathrm{mcg} / \mathrm{L}$ order to secure the ruptured aneurysm or decompress the injured brain [13]. Non-pharmacological strategies include early mobilization and intermittent pneumatic compression devices that should be started as soon as possible after hospitalization; however, current guidelines recommend the association of PT to lower DVT risk at least $24 \mathrm{~h}$ after SAH [13]. Despite guidelines, many clinicians are reluctant to start anticoagulants in these patients and many centers initiate the pharmacological prophylaxis toward the end of the ICU stay [17]. In our institution, it is standard practice to initiate thromboprophylaxis with intermittent pneumatic compression immediately after hospitalization, whereas enoxaparin at prophylactic dosage (4000 IU) is typically initiated $48 \mathrm{~h}$ after the acute event except for patients with pending surgical procedures. Rebleeding after the initiation of PT was very rare, with only one patient out of 170 presenting a non-significant rebleeding of the intracranial hematoma.

Identification of predictors for DVT might be useful in determining the cohort of patients which can benefit the most from an in-depth examination and may help us determine which category of patients requires an early venous duplex ultrasound, simplifying the screening process and making it both more cost-effective and less time consuming.

According to the results of our study, d-dimer levels at the time of hospitalization and the presence of an $\mathrm{ICH}$ can be predictors of DVT in patients admitted for SAH.

Recent findings suggest that establishing a d-dimer protocol in the general neurosurgical population may be beneficial for the purpose of reducing venous duplex ultrasound [6]. Furthermore, our results are in accordance with those of a study on Chinese patients with $\mathrm{ICH}$ which suggested that increased d-dimer level poses an increased risk of developing DVT [3]. In our study, we confirm this finding on the SAH population analyzed and demonstrate that the presence of $\mathrm{ICH}$ as well as elevated d-dimer levels may increase the likelihood of DVT. The presence of IVH, on the other hand, was not associated with DVT in both univariate analysis ( $p$ value 0.833 ) and logistic regression ( $p$ value: 0.986 ).

Motor deficit, a well-established risk factor, is frequently used in scores to evaluate the risk of deep-vein thrombosis [19] and is confirmed as an important clinical tool in the assessment of patients with suspect DVT. On the other hand, it is often discovered in the later stages of the ICU stay when sedation is reduced, meaning it may be difficult to adopt it in a screening strategy.

A high d-dimer level is common in patients with SAH [20], making its clinical use difficult as a standalone predictor marker for DVT risk. In our study, the median d-dimer level at admission was generally increased $(613 \mathrm{mcg} / \mathrm{L})$ similarly among patients with or without ICH. Nonetheless, in patients with ICH who later developed DVT, d-dimer levels were generally significantly increased (median $849 \mathrm{mcg} / \mathrm{L}$ ). 
ROC curve for d-dimer, motor deficit, and ICH revealed a slightly higher AUC than the ROC curve for d-dimer and ICH (AUC was increased by 0.004 compared to ROC for d-dimer and ICH). Considering the negligible increase in AUC with the addition of motor deficit to the model, we believe that the presence of $\mathrm{ICH}$ and d-dimer level at hospitalization may represent an interesting tool for DVT prediction; however, such a tool needs to be prospectively validated.

Different possible explanation can be formulated to justify these findings: early activation of the coagulation cascade derived from a bleeding associated with the damage to the blood brain barrier in the context of an intraparenchymal hemorrhage may be associated with an increased level of d-dimer, a marker of fibrin formation and reactive fibrinolysis, in the early phase of the disease. The increase in d-dimer in this context may reflect a potent early activation of the coagulation cascade. A hypercoagulable state measured through thromboelastography has been demonstrated in rats after induced SAH [8], and possible explanations include the release of tissue factor (TF), abundantly present in the brain tissue [2], which initiates the coagulation cascade. The release of TF into the systemic blood circulation leads to the activation of thrombin, which in turn promotes the formation of fibrin and platelet aggregations in the damaged vessel, a fundamental process in preventing further hemorrhage $[1,14]$. It has been demonstrated that there is a relative imbalance between the levels of TF and tissue factor plasminogen inhibitor (TFPI) in patients with SAH and DVT when compared to patients with SAH and no DVT [11]. This imbalance may partially explain why d-dimer levels increase in greater proportion in DVT patients when compared to non-DVT patients. We hypothesize that the abundance of TF released during the bleeding event promotes a relative hyperactivation of the coagulation system to counter the hemorrhage, with greater formation of fibrin, and thus a greater release of fibrinogen degradation products, including d-dimer. The aneurysm generally ruptures and inundates the subarachnoid space with blood and the subarachnoid hemorrhage may extend to the parenchymal space: it is reasonable to assume that the release of tissue factor and the resulting mechanism of initial hypercoagulability is greater in this instance than when the hemorrhage is confined to the subarachnoid space, but further research is required to prove this hypothesis.

Our study has some limitations that need to be discussed.

A potential bias is related to our limited sample size. Patients included in this study were retrieved only from the EMR of our institution, thus limiting the sample.

In order to properly assess which patients can benefit from a more aggressive screening strategy or a tailored approach to pharmacoprophylaxis, we believe further research including parameters such as symptomatic deepvein thrombosis, cases of pulmonary embolism, and associated mortality are needed.

\section{Conclusion}

Elevated d-dimer levels upon hospitalization, motor deficit, and the presence of intraparenchymal hemorrhage are independent risk factors for DVT. Patients with elevated d-dimer and concomitant intraparenchymal cerebral hemorrhage may benefit from a more aggressive screening strategy for DVT. To the best of our knowledge, this is the first study describing concomitant intraparenchymal cerebral hemorrhage in the setting of SAH as a potential risk factor for DVT.

\section{Compliance with ethical standards}

Conflict of interest The authors declare that they have no conflict of interest.

Ethical approval All procedures performed in studies involving human participants were in accordance with the ethical standards of the institutional and/or national research committee and with the 1964 Helsinki declaration and its later amendments or comparable ethical standards.

For this type of study, formal consent is not required.

\section{References}

1. Brass LF (2003) Thrombin and platelet activation. Chest 124:18S25S. https://doi.org/10.1378/chest.124.3 suppl.18s

2. Butenas S, Orfeo T, Brummel-Ziedins KE, Mann KG (2007) Tissue factor in thrombosis and hemorrhage. Surgery 142:S2S14. https://doi.org/10.1007/s00701-010-0699-1

3. Cheng X, Zhang L, Xie NC, Ma YQ, Lian YJ (2016) High plasma levels of D-dimer are independently associated with a heightened risk of deep vein thrombosis in patients with Intracerebral hemorrhage. Mol Neurobiol 53:5671-5678. https://doi.org/10.1007/ s12035-015-9487-5

4. Francoeur CL, Mayer SA (2016) Management of delayed cerebral ischemia after subarachnoid hemorrhage. Crit Care 20:277. https:// doi.org/10.1186/s13054-016-1447-6

5. Huang J, van Gelder JM (2002) The probability of sudden death from rupture of intracranial aneurysms: a meta-analysis. Neurosurgery 51:1101-1105. https://doi.org/10.1097/00006123200211000-00001

6. Karsy M, Azab MA, Harper J et al (2019) Evaluation of a D-dimer protocol for detection of venous thromboembolism. World Neurosurg. https://doi.org/10.1016/j.wneu.2019.09.160

7. Kshettry VR, Rosenbaum BP, Seicean A, Kelly ML, Schiltz NK, Weil RJ (2014) Incidence and risk factors associated with inhospital venous thromboembolism after aneurysmal subarachnoid hemorrhage. J Clin Neurosci 21:282-286. https://doi.org/10.1016/j. jocn.2013.07.003

8. Larsen CC, Hansen-Schwartz J, Nielsen JD, Astrup J (2010) Blood coagulation and fibrinolysis after experimental subarachnoid hemorrhage. Acta Neurochir 152:1577-1581. https://doi.org/10.1007/ s00701-010-0699-1

9. Mack WJ, Ducruet AF, Hickman ZL et al (2008) Doppler ultrasonography screening of poor-grade subarachnoid hemorrhage patients increases the diagnosis of deep venous thrombosis. Neurol Res 30:889-892. https://doi.org/10.1179/174313208X327946

10. Malato A, Dentali F, Siragusa S (2015) The impact of deep vein thrombosis in critically ill patients: a meta-analysis of major clinical 
outcomes. Blood Transfus 13(4):559-568. https://doi.org/10.2450/ 2015.0277-14

11. Miao W, Zhao K, Deng W, Teng J (2018) Coagulation factor Hyperfunction after subarachnoid hemorrhage induces deep venous thrombosis. World Neurosurg 110:e46-e52. https://doi.org/10. 1016/j.wneu.2017.09.200

12. Moussouttas M, Bhatnager M, Huynh TT et al (2013) Association between sympathetic response, neurogenic cardiomyopathy, and venous thromboembolization in patients with primary subarachnoid hemorrhage. Acta Neurochir 155:1501-1510. https://doi.org/10. 1007/s00701-013-1725-x

13. Nyquist P, Bautista C, Jichici D (2016) Prophylaxis of venous thrombosis in neurocritical care patients an evidence-based guideline: a statement for healthcare professionals from the neurocritical care society. Neurocrit Care 24:47-60. https://doi.org/10.1007/ s12028-015-0221-y

14. Peltonen S, Juvela S, Kaste M, Lassila R (1997) Hemostasis and fibrinolysis activation after subarachnoid hemorrhage. J Neurosurg 87:207-214. https://doi.org/10.3171/jns.1997.87.2.0207

15. Ray WZ, Strom RG, Blackburn SL, Ashley WW, Sicard GA, Rich KM (2009) Incidence of deep venous thrombosis after subarachnoid hemorrhage. J Neurosurg 110:1010-1014. https://doi.org/10. 3171/2008.9.JNS08107
16. Rowland MJ, Hadjipavlou G, Kelly M, Westbrook J, Pattinson KT (2012) Delayed cerebral ischaemia after subarachnoid haemorrhage: looking beyond vasospasm. Br J Anaesth 109:315-329. https://doi.org/10.1093/bja/aes264

17. Sauro KM, Soo A, Kramer A et al (2019) Venous thromboembolism prophylaxis in neurocritical care patients: are current practices, best practices? Neurocrit Care 30:355-363. https://doi.org/10.1007/ s12028-018-0614-9

18. Terpolilli NA, Brem C, Bühler D, Plesnila N (2015) Are we barking up the wrong vessels? Cerebral microcirculation after subarachnoid hemorrhage. Stroke 46:3014-3019. https://doi.org/10.1161/ STROKEAHA.115.006353

19. Wells PS, Hirsh J, Anderson DR et al (1995) Accuracy of clinical assessment of deep-vein thrombosis. Lancet 345(8961):1326-1330

20. Zhang Y, Yang P, Wang J (2019) Diagnostic value of D-dimer in distinguishing between spontaneous subarachnoid hemorrhage and nontraumatic acute headache. Clin Lab 1:65(11). https://doi.org/10. 7754/Clin.Lab.2019.190230

Publisher's note Springer Nature remains neutral with regard to jurisdictional claims in published maps and institutional affiliations. 